\title{
Publishing is evolving, but are we? Developing talent for the future
}

\author{
Andrew James Miller* \\ Organizational Coach, Taylor \& Francis Group, Oxford, UK
}

\begin{abstract}
At the APE 2020 Pre-Conference in Berlin, a group of talent development and HR experts from across the scientific research and publishing community came together to discuss the future of talent development in the scholarly publishing industry.

We heard from an excellent group of speakers who shared with us a rich and diverse range of expertise and experience. We set ourselves the challenge of imagining what the world of scholarly academic publishing would look like in 2030, and asked ourselves the question: how can we work together to develop the talent we will need now, and for the future, in a rapidly changing world? Are we keeping pace, and are we prepared for the challenge ahead?

Based on our discussion, three key themes emerged: the importance of supporting increased diversity \& inclusion within scholarly publishing, interorganizational leadership development initiatives for leaders across the publishing ecosystem to exchange experiences and ideas, and greater research and publishing career mobility to encourage more fluid movement between research and publishing jobs.

We believe these things are all very achievable if we commit to investing in the kind of culture change and new ways of thinking that will lead us to success in 2030.
\end{abstract}

Keywords: Research, scholarly publishing, academia, talent development, diversity \& inclusion, leadership development, career mobility, change management, culture

At the APE 2020 Pre-Conference we heard from a truly excellent group of speakers who shared with us a rich and diverse range of expertise and experience. We set ourselves the challenge of imagining what the world of scholarly academic publishing would look like in 2030, and asked ourselves the question: how can we work together to develop the talent we will need now, and for the future, in a rapidly changing world? Are we keeping pace, and are we prepared for the challenge ahead?

So in what ways is our world changing? From our Speakers and group discussions we gained an exciting insight into...

A world where the expectation is that content must be free, and easily accessible, immediately when we want to consume it. The expert curators are our social networks, recommending, sharing, liking and creating playlists based on our shared interests and talking points. Learners now plot their own learning path, self-directing their learning to get the knowledge and information they need as and when they need it.

A world where technology is increasingly intelligent, able to have natural language conversations with us, and to solve real-world problems for us. Digital technology has evolved from being a virtual "tool" that we use to aid us in completing a task, to a virtual "Assistant" we can rely on to do the task for us, even able to learn and teach itself new skills.

*E-mail: Andrew.Miller@TandF.co.uk. 
A world where organizations are truly global, with geographical boundaries to teamwork and collaboration now a thing of the past, decentralized in their decision-making through flatter structures, and freed from the necessity of physical office environments. Driven by data, big and small, to inform their product development strategies and performance metrics, and empower decision-making.

And finally, a world where talent is measured increasingly in "soft" skills, or rather "power skills" as we renamed them, in recognition of the importance of emotional intelligence and creativity alongside technical skills. The "gig economy" has seen talent become increasingly mobile and on-demand, changing the nature of careers and employer relationships, and the type of talent and specialist skills we need is evolving at a rapid rate, making the sourcing and development of talent a continuous process of evolution.

This is all against the backdrop of shifting social values towards an increased focus on diversity \& inclusion, sustainability and ethical business practices.

We discussed and debated the challenges and opportunities that have emerged, and are emerging, as a result of these changes, with great optimism and with the benefit of a number of exciting case studies of how the scholarly publishing community has embraced and capitalized on many of these new trends. Though we all agreed that even greater change was still to come.

We did however also talk about the challenges that inevitably come in times of change, and how the organizational change required to keep pace with these challenges, is never easy.

As we discussed some of the key difficulties we are all facing, it became apparent that for an organization to achieve successful and meaningful change, it would have to work against the very structures that hold it together.

Organizations are built around a tightly controlled structure: processes, procedures, policies, hierarchies, established ways of working. Organizations are designed to succeed through establishing, and maintaining, order. Is it any wonder that they, and their people, are resistant to change?

Organizations typically reward, incentivize and celebrate success, and punish failure. Is it any wonder, therefore, that people fear failure, when it comes to experimenting and taking on the risks associated with change?

Organizations most commonly reward individual achievement, through bonus structures and promotions. When we ask for increased collaboration and teamwork to take on the challenges of change, does our organization incentivize the investment of time and energy put into that collaboration above individual achievement?

The kind of change we need, therefore, goes deep, and the first things we need to focus on are the things holding us back from being able to change.

The heart and soul of an organization is its culture. We felt that the extent of the changes we are all faced with in the worlds of content, technology, organizations, and talent, and the pace of those changes, will require a depth of change that can only come from "doubling the loop", to draw inspiration from Kolb's famous model [1], and asking ourselves the hard questions about our own organizational cultures.

A question perhaps for all of us here today: within our own organizations, how much attention, energy, and resources have we put into to new strategies, and how much, comparatively into culture change?

So, to borrow a thought from Albert Einstein, "we cannot solve our problems with the same thinking we used when we created them." Could it be that we need to adopt a different kind of thinking? What does that mean, and what does that look like?

Based on our discussion from the Pre-Conference, three ideas emerged that I would like to share with you:

1. Diversity \& inclusion was a key theme of the pre-conference. The research shows that diverse organizations are more innovative, more adaptive, and more successful, and we heard a number of 
inspiring success stories from our Speakers and our group discussions. But we all agreed that we could do more to support all types of diversity, as something that requires sustained effort and leadership, and that should be front and center of any meaningful cultural change within an organization, and within an industry.

2. We all agreed that the best thing about the pre-conference day was the opportunity to share experiences and ideas with people from different organizations across the publishing ecosystem. The collective goodwill, energy and creativity created a buzz that reminded us that if our industry becomes stronger, we all become stronger. There was also recognition that good leadership is something that will never lose importance, and that a key part of any leader's development should be the broadening of their horizons and the sharing of perspectives, experience and insights with leaders across their industry, not just within their own organization. So we believe that another key component to successful change can be found in interorganizational leadership development initiatives for leaders across the publishing ecosystem, to get together, and develop together.

3. We also talked a lot about careers, and the development of talent from early-career onwards, and how in a world where careers no longer follow a simple linear progression, it was important to encourage professionals to think laterally about their career path. Publishing and research are still largely seen as two separate career tracks - yet they are so interlinked and symbiotic - what can we do to break down the boundaries and encourage more fluid movement between research and publishing jobs and roles? What would allow for greater knowledge-sharing, skills transfer and offer more opportunities for development, career advancement, and growth, than the opportunities of that "cross-fertilization", as we called it? Placements, internships, the way we talk about careers in research and publishing - these are all opportunities that we think can be explored.

We believe that these three things would greatly benefit all our organizations, and are all very achievable, if we are committed to investing in the kind of culture change and new ways of thinking that we believe will lead us to the exciting and successful future that we envision for 2030.

A final thought: in the pre-conference we talked about the importance of individual accountability, and that every one of us can contribute in a profound, meaningful way, to creating the future we want to see.

I would like you to turn to the person seated next to you.

For the next 5 minutes, I would like you to discuss how you individually could make a meaningful contribution to your organization taking positive steps in any of these three areas:

(1) Diversity in scholarly publishing

(2) Interorganizational leadership development

(3) Research and Publishing career mobility

If you like, you can make a commitment to each other about something you plan to do.

Thank you.

\section{References}

[1] D.A. Kolb, Experiential Learning: Experience as the Source of Learning and Development, 1st ed. Prentice Hall, London, 1984. 